\title{
An Etiological Study of Dilated Cardiomyopathy in Correlation with Clinical, ECG And Echocardiographic Profile
}

\author{
Dr. Divakar Kumar ${ }^{1}$,Dr. Vidyapati ${ }^{2}$,Dr. M. L. Prasad ${ }^{3}$,Dr. Manoj Kumar ${ }^{4}$ \\ ${ }^{1}$ Senior Resident, Department of Medicine, RIMS Ranchi \\ ${ }^{2}$ Associate professor, Department of Medicine, RIMS Ranchi \\ ${ }^{3}$ Assistant professor, Department of Medicine, RIMS Ranchi \\ ${ }^{4}$ Assistant professor, Department of Medicine, RIMS Ranchi
}

\begin{abstract}
Cardiomyopathy is a primary disorder of the heart muscle that causes abnormal myocardial performance and is not the result of disease or dysfunction of other cardiac structures. Dilated cardiomyopathy is the most common variety of cardiomyopathy. Other types of cardiomyopathy are hypertrophic and restrictive cardiomyopathy. Dilated cardiomyopathy represents the final common pathway produced by a variety of ischaemic, toxic, metabolic and immunological mechanisms damaging the heart muscle. The incidence of DCM is reported to be 5 to 8 cases per 1,00,000 population per year. DCMP is more common in male and blacks. Natural history of DCM is not well established. The patient can present with variable symptoms like heart failure, arrhythmias, stroke or sudden death. Some may have minimal or no symptoms. The present study aims to evaluate the etiological, electrocardiographical, echocardiographical and clinical profile of DCMP patients. The present study is one year cross sectional study on patients admitted in the department of Medicine, RIMS Ranchi. Patient was included based on Clinical and Echocardiographic criteria while those with Valvular and Congenital heart disease were excluded. Statistical analysis was done with percentage, proportions, chi-square and correlation. The results of this study showed majority of patients were males compromising $56.6 \%$ and females were $43.25 \%$. In males DCMP was mostly seen in elderly while in females it was mostly seen in middle age. The most common etiology was ischaemic DCMP comprising $33.3 \%$ patients, followed by diabetic, peripartum, idiopathic and alcoholic. The most common presentation in our study was biventricular failure which was seen in $80 \%$ of cases followed by isolated Left ventricular failure. Majority of patients were in NYHA class IV and III. Other symptoms were cough, easy fatiguability, pedal edema, abdominal pain, palpitation, chest pain and syncope. ECG findings included QRS axis deviation (left and right), ventricular ectopics, sinus tachycardia, LBBB, RBBB, AF, atrial ectopics, SVT, VT, CHB, LVH and nonspecific ST-T changes. Echocardiographic findings included reduced LV ejection fraction, $M R, T R, L V$ clot and pericardial effusion. Chest radiography findings included increased cardiothoracic ratio, pleural effusion and pulmonary plethora. Conclusion of the study included most common type is DCMP, most common etiology is ischaemic and most common clinical presentation is biventricular failure. The most common ECG findings were sinus tachycardia and ventricular ectopics. In Echocardiography most common finding was reduced Left ventricular ejection fraction.
\end{abstract}

Keywords: FNAC, USG, HPE, Thyroid Nodule.

\section{Introduction}

Cardiomyopathy is a primary disorder of the heart muscle that causes abnormal myocardial performance and is not the result of disease or dysfunction of other cardiac structures. The dominant feature is a direct involvement of the heart muscle itself. The 1980 WHO committee reserved the term cardiomyopathy for myocardial disease of unknown cause. The most widely used functional classification of cardiomyopathy recognizes disturbances of function-dilatation, hypertrophy and restriction. Dilated cardiomyopathy is the most common variety of cardiomyopathy (Richardson, 1980). Current diagnosis and treatment of DCMP varies somewhat among the various types, but the cornerstone of medical management are similar in most cases. The most common dilated cardiomyopathy is ischaemic DCMP followed by idiopathic, familial, diabetic and alcoholic (Richardson, 1980). Dilated cardiomyopathy represents the final common pathway produced by a variety of ischemic, toxic, metabolic, and immunologic mechanisms damaging the heart muscle. The most common clinical presentation is heart failure, usually left ventricular failure. The patient can also present with symptoms secondary to arrhythmias, stroke (embolic infarction) or sudden death. The prevalence of heart failure is about 1 to $1.5 \%$ of the adult population. The mortality and morbidity remains high (median survival of 1.7 year for men and 3.2 year for women). DCMP is an important cause of heart failure and accounts for upto $25 \%$ of all cases of heart failure. The incidence and prevalence of heart failure due to cardiomyopathy appears to be increasing. The incidence of DCMP is reported to be 5 to 8 per 1 lakh population per year. It occurs 3 times more frequently in males as compared to females. It is also more common in blacks. The natural history of 
DCM is not well established. Many patient have minimal or no symptoms and the progression of the disease is unpredictable. The long term prognosis is not good. Nevertheless, in symptomatic patients the course is usually one of progressive deterioration with upto $50 \%$ of patients with heart failure succumbing within a year. The annual mortality rate for a typical patient of DCM with heart failure is about 11 to 13 percent. Exact epidemiology data on DCMP in India are lacking. With the rapid advancement in molecular genetics and uncovering of underlying etiologies, DCMP is being recognized as a specific diagnosis and not one of exclusion. In view of the high prevalence of chronic heart failure due to underlying DCMP and the lack of data on DCMP, this study was undertaken.

\section{Objectives}

To study the etiological, clinical, electrocardiographic and echocardiographic profile of patients. Incidence of dilated cardiomyopathy in relation to other types of cardiomyopathies in patients admitted in RIMS Ranchi.

\section{Materials And Methodology}

This study was done on patients admitted with symptoms and signs of heart failure in medical ward of Department of Medicine, RIMS, Ranchi over a period of one year. This was a cross sectional study and included 30 patients who fulfilled the inclusion criteria of clinical and echocardiographic findings. Clinical findings included Symptoms and Signs of heart failure while echocardiographic findings included Left ventricular ejection fraction $<45 \%$, Left ventricular end diastolic dimension $>3 \mathrm{~cm} /$ body surface area, global hypokinesia and dilatation of all the chambers of heart. The patients with valvular and congenital heart diseases were excluded from study. Other investigations done was chest radiography, coronary angiography, CBC, Blood glucose, HbA1c, liver function tests, etc. statistical analysis was done with percentage, proportions, chi-square and correlation.

\section{Results}

Table - I: Etiological distribution of DCMP in the present study

\begin{tabular}{|c|c|c|}
\hline Cardoimyopathy & Number & Percentage \\
\hline Ischemic & 10 & 33.3 \\
\hline Idiopathic & 4 & 13.3 \\
\hline Diabetic & 7 & 23.3 \\
\hline Peripartum & 5 & 16.6 \\
\hline Alcoholic & 2 & 6.6 \\
\hline Miscellaneous & 2 & 6.6 \\
\hline
\end{tabular}

The most common type of DCMP was ischaemic DCMP comprising $33.3 \%$ of all followed by diabetic $(23.3 \%)$ and peripartum (16.6) DCMP. The other types were idiopathic and alcoholic. Miscellaneous group included $\beta$-thalassemia intermedia and polymyositis. In the Demographic profile of patients with DCMP, majority of the patients were above the age of 60 years of which males comprised $56.66 \%$ and females comprised $43.25 \%$. Majority of cases among males were above the age of 60 years while in females there was clustering of cases among young adults and middle aged population. Some females were below 19 years.

Table - II: Symptom profile in the present study

\begin{tabular}{|c|c|c|}
\hline Symtoms & Number & Percentage \\
\hline Dyspnea & 30 & 100 \\
\hline Palpitation & 17 & 56.6 \\
\hline PND & 18 & 60 \\
\hline Orthopnea & 16 & 53.3 \\
\hline Chest pain & 12 & 40 \\
\hline Pedal edema & 21 & 70 \\
\hline Cough & 18 & 60 \\
\hline Abdominal pain & 10 & 33.3 \\
\hline Easy fatiguability & 25 & 83.3 \\
\hline Syncope & 5 & 16.6 \\
\hline Asymptomatic & None & 0 \\
\hline Miscellaneous & 7 & 23.3 \\
\hline
\end{tabular}

All the patients presented with exertional dyspnea. Easy fatiguability was seen in $83.3 \%$ of subjects, the second most common symptom followed by pedal edemain $70 \%$ of patients. Other symptoms were PND, cough, palpitation, orthopnea, chest pain, abdominal pain and syncope. 
Table - III: NYHA class in the present study

\begin{tabular}{|c|c|c|}
\hline NYHA class & Number & Percentage \\
\hline Class I & 1 & $3.3 \%$ \\
\hline Class II & 5 & 16.6 \\
\hline Class III & 10 & 33.3 \\
\hline Class IV & 14 & 46.6 \\
\hline
\end{tabular}

Majority of the patients were in NYHA class III (33\%) and class IV (46\%) group

Table - IV: Types of Heart failure in the present study

\begin{tabular}{|c|c|c|}
\hline Compartment involved & Number & Percentage \\
\hline LVF & 5 & 16.6 \\
\hline RVF & 1 & 3.3 \\
\hline Biventricular & 24 & 80 \\
\hline
\end{tabular}

Biventricular failure was seen in $80 \%$ of patients. Isolated LV failure was seen in $16.6 \%$ and RV failure in 3.3\%.In the present study patients has different physical signs. Basal crepitations were seen in almost $93.33 \%$ of total patients. Pedal edema was present in $76.6 \%$. Raised JVP was seen in $73.3 \%$ and hepatomegaly in $46.6 \%$. Other signs were Apical pansystolic murmur, third heart sound and pansystolic murmur in tricuspid area. Systolic blood pressure $<100 \mathrm{mmHg}$ was seen in $26.6 \%$ and one patient had stroke. Abnormalities of peripheral pulses included ectopic beats in $53.3 \%$ of patients, tachycardia in $46.6 \%$ and atrial fibrillation in $13.3 \%$ of patients. Bradycardia and pulsus alternans were other abnormalities.

In the chest radiography cardiomegaly was seen in all the patients. Cardiothoracic ratio was more than 0.7 in $13.3 \%, 0.6$ to 0.4 in $40 \%$ and mild cardiomegaly i.e. between 0.5 to 0.6 in $46.6 \%$ of patients. Pulmonary plethora was seen in $53.3 \%$ while pleural effusion was seen in $20 \%$ of patients.

Table - V: Electrocardiographic profile of patients in the present study

\begin{tabular}{|l|l|c|c|}
\hline \multicolumn{1}{|c|}{ Parameters } & \multicolumn{1}{|c|}{ Normal } & Percentage \\
\hline QRS axis & \multicolumn{1}{|c|}{ Normer } & 80 \\
& Left axis deviation & 4 & 13.3 \\
& Right axis deviation & 2 & 6.6 \\
\hline Arrhythmias & Sinus tachycardia & 12 & 40 \\
& Atrial ectopics & 3 & 10 \\
& Atrial fibrillation & 4 & 13.3 \\
& SVT & 2 & 6.6 \\
& Ventricular ectopics & 14 & 46.6 \\
& Ventricular Tachycardia & 1 & 3.3 \\
& Complete heart block & 1 & 3.3 \\
& Left bundle branch block & 12 & 40 \\
& Right bundle branch & 4 & 13.3 \\
& block & 8 & 26.6 \\
\hline ST-T changes & & 4 & 13.3 \\
\hline Atrial enlargement & LAE & 2 & 6.6 \\
\hline Ventricular & RAE & 6 & 20 \\
hypertrophy & LVH & 2 & 6.6 \\
& RVH & 1 & 3.3 \\
\hline
\end{tabular}

The electrocardiographic profile included abnormalities of rate, rhythm, axis and chamber enlargement. The most common abnormality was ventricular ectopics seen in $46.6 \%$ of patients. Sinus tachycardia and left bundle branch blocks were seen in $40 \%$ of patients. RBBB was observed in $13.3 \%$ of patients. Other ECG abnormalities were NonSpecific ST-T changes, atrial fibrillation, LVH and left and right atrial enlargement. The axis was normal in majority. Left axis deviation was seen in $13.3 \%$ and right axis deviation in $6.6 \%$.

Table - VI: Echocardiographic profile of patients in the present study

\begin{tabular}{|l|l|l|l|}
\hline Parameters & Range & Number & Percentage \\
\hline Ejection fraction & $40-45 \%$ & 5 & 16.6 \\
& $30-39 \%$ & 11 & 36.6 \\
& $20-29 \%$ & 12 & 40 \\
& $<20 \%$ & 2 & 6.6 \\
\hline LVEDD & $4.5-4.9 \mathrm{~cm}$ & 4 & 13.3 \\
& $5.0-5.9 \mathrm{~cm}$ & 10 & 33.3 \\
& $>6 \mathrm{~cm}$ & 16 & 53.3 \\
\hline LVSD & $3.5-4 \mathrm{~cm}$ & 6 & 20 \\
& $4.0-4.9 \mathrm{~cm}$ & 10 & 33.33 \\
& $>5 \mathrm{~cm}$ & 14 & 46.6 \\
\hline
\end{tabular}


An Etiological Study Of Dilated Cardiomyopathy In Correlation With Clinical, ...

\begin{tabular}{|l|l|l|l|}
\hline Mitral regurtitation & & 21 & 73.3 \\
\hline $\begin{array}{l}\text { Tricuspid } \\
\text { regurtitation }\end{array}$ & 3 & 10 \\
\hline Pericardial effusion & & 2 & 6.6 \\
\hline
\end{tabular}

The mean LV ejection fraction was $30.87 \%$. The left ventricular ejection fraction was less than $20 \%$ in $6 \%$ of patients. It was between $20-29 \%$ in $40 \%$, between $30-39 \%$ in $36.6 \%$ of patients and between $40-45 \%$ in $16.6 \%$ of patients. The mean LV end diastolic diameter was $5.86 \mathrm{~cm}$ while the mean LV end systolic diameter was $4.75 \mathrm{~cm}$. Global hypokinesia and dilatation of all 4 chambers were seen in all the patients. In our study $73.3 \%$ had mitral regurgitation, $10 \%$ had tricuspid regurgitation and pericardial effusion was seen in $6 \%$ of patients.

\section{Discussion}

The present study aims to evaluate the etiological, clinical, electrocardiographic and echocardiographic profile of the patients and also the incidence of dilated cardiomyopathy in relation to other types of cardiomyopathies in patients admitted in RIMS, Ranchi. In our study the mean age of presentation in males was 56.88干15.99 years while in females DCM was predominantly seen in middle age (41.15 $\mp 20.19$ years). This data corresponds to study by Ahmad S in 2005. In our study few female patients were of age below 19 years. In our study the most common type of DCM was ischaemic DCM in $33.3 \%$ of patients followed by diabetic in $23.3 \%$ and peripartum DCM in $16.6 \%$ of patients. Other types were idiopathic, alcoholic and miscellaneous which included $\beta$-thalassemia intermedia and polymyositis. These percentages were similar to study by Jain et al. Coronary angiography was done in all patients with ischaemic DCM. Of the 10 patients studies 6 had history of previous MI. All the 10 patients had significant narrowing of epicardial coronaries (i.e. $>70 \%$ of lumen) six patients had double vessel disease, three showed triple vessel disease and one had single vessel disease. The echocardiography of all the patients showed global hypokinesia with reduced ejection fraction. Liver function tests revealed mildly raised bilirubin $(1.5 \mathrm{mg} \%)$ with normal liver enzymes. This is similar to that found in other studies on alcoholic cardiomyopathy. In our study $33.3 \%$ of patients had anemia, most of the patients had mild anemia ( $\mathrm{Hb} \%$ 8.5-11 gm) which is similar to study by A. Justin et al. The most common presentation in our study was biventricular failure seen in $80 \%$ of cases followed by isolated LV and RV failure. Majority of the patients were in NYHA class IV (46.6\%) and class III $(33.3 \%)$. Breathlessness was the commonest symptom noticed in all patients followed by PND and orthopnea. Other symptoms were cough, easy fatiguability, pedal edema, abdominal pain, palpitation, chest pain and syncope. Physical signs included basal crepitations, LVS3, RVS3, murmurs of MR and TR, raised JVP, hepatomegaly, tachycardia, bradycardia, ectopics and other findings. Presentation with biventricular failure and symptoms of exertional dyspnea, easy fatiguability, pedal edema, cough, palpitation and abdominal pain were similar to that of study by Ahmad S in 2005, Singh G in 2002 and Jain A in 2004. In our study $40 \%$ of patients had chest pain while $16.6 \%$ had syncope which is different from study by S. Ahmed et al. because they had excluded patients of ischaemic DCMP. In our study the most common ECG finding was sinus tachycardia similar to study by Ahmed et al. The QRS axis presentation and non specific ST-T changes were similar to other studies. LVH was less commonly seen in our study (20\%) compared to $30-40 \%$ in other studies.

However RBBB, CHB and SVT were more commonly present in our study in comparison to other studies which could be due to inclusion of ischemic cardiomyopathy in our study. The mean LV ejection fraction in our study group was $30.87 \%$, similar to other studies. The mean LV end diastolic and systolic diameter were less compared to other studies. However fractional shortening was comparable to all the other groups. MR was more common than TR in our study because of large portion of patients of ischaemic DCM compared to nonischaemic DCM. None of our patients had AR compared to $17.8 \%$ of patients in Jain et al study. MR and TR in DCM are secondary to annular ring dilatation. Left ventricular clot seen in one patient who also had cerebral embolism secondary to AF. Pericardial effusion was present in $6.6 \%$ of our patients. Chest radiography findings included cardiomegaly which was similar in percentage to study by Massumi et al. while pleural effusion and pulmonary plethora was present in less number of patients compared to study by Massumi et al. and Ahmed et al.

\section{The conclusion of the study were:}

\section{Conclusion}

Dilated cardiomyopathy is the most common type of cardiomyopathy and an important cause of congestive heart failure. DCMP is common in elderly and middle aged population. It is more common in males. The most common clinical presentation is biventricular failure followed by left ventricular failure. The most common type is ischemic DCM followed by diabetic, peripartum, idiopathic and alcoholic DCM. Ischaemic DCM was the most common subtype in males while idiopathic and peripartum DCM in females. chest radiograph showed cardiomegaly in most patients. The common abnormalities on ECG consist of sinus tachycardia, atrial fibrillation and left bundle branch block. Echocardiography revealed reduced ejection fraction 
and global hypokinesia universally. Mitral regurgitation and pericardial effusion were present in significant number of patients. Ejection fraction correlated well with NYHA class. Most common clinical presentation is dyspnea, easy fatiguability and pedal edema. Edema is presentation of the cardiac, renal and liver diseases but if any elderly male patient presenting with dyspnea, easy fatiguability and pedal edema and female patient of middle age group is presenting with symptom then DCMP should be suspected and early treatment will give better results.

\section{References}

[1]. Richerdson. WHO report on classification of cardiomyopathy Br. Heart J. 1980; 44: 680-682

[2]. Framingham heart study nih.gov/Framingham

[3]. Braunwald and Bristow MR. Congestive heart failure: fifty years of progress. Circulation 2000; $102 ; 14$

[4]. Hurst's The heart classification of cardiomyopathy. Chap 75: 1886-1887

[5]. Ahmad S, Rabbani M. clinical ECG and echocardiographic profile of patients with dilated cardiomyopathy. Indian J Cardiol 2005; 8: $25-29$

[6]. Singh G, Nayyar SB. Clinical profile of dilated cardiomyopathy - A study of 138 cases. JAPI2002: 50: 1156

[7]. Jain A, Tewari S clinical profile of dilated cardiomyopathy. Indian Heart J 2004; 56: 507-17

[8]. Massumi RA, Jorge CR. Primary myocardial disease Report of 50 cases and review of the subject. Circulation 1965; 31: 19-40 